CLINICAL AND EXPERIMENTAL VACCINE RESEARCH

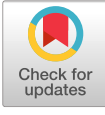

Clin Exp Vaccine Res 2020;9:56-63 https://doi.org/10.7774/cevr.2020.9.1.56 pISSN 2287-3651 • elSSN 2287-366X

Yong Wook Park ${ }^{1,2}$, Yun Hee Kim', Hwan Ui Jung ${ }^{1}$, Oh Seok Jeong', Eun Ji Hong', Hun Kim', Jae II Lee ${ }^{2}$ 'Department of Bio R\&D, SK Bioscience, Seongnam; ${ }^{2}$ Department of Veterinary Medicine, College of Veterinary Medicine, Chonnam National University, Gwangju, Korea

Received: January 10, 2020

Revised: January 20, 2020

Accepted: January 21, 2020

Corresponding author: Yong Wook Park Department of Bio R\&D, SK Bioscience, 310 Pangyo-ro, Bundang-gu, Seongnam, 13494, Korea Tel: +82-2-2008-3923, Fax: +82-2-2008-2047 E-mail: ifloat@sk.com

No potential conflict of interest relevant to this article was reported.

This study was performed with SK Bioscience's financial supports.

\section{Comparison of antigenic mutation during egg and cell passage cultivation of H3N2 influenza virus}

Purpose: When influenza viruses are cultured in eggs, amino acid mutations of the hemagglutinin may occur through egg adaptation. On the other hand, when influenza viruses are cultured in animal cells, no antigenic mutation occurs unlike in eggs. Therefore, we examined whether the antigenic mutations actually occurred after passage of H3N2 (A/Texas/50/2012) virus up to 15 times in eggs and MDCK-Sky3851 cells.

Materials and Methods: Prototype A/Texas/50/2012 (H3N2) influenza virus which was isolated from clinical patient, not passaged in egg, was obtained and propagated using the specific pathogen free egg and the MDCK-Sky3851 cell line up to 15 passage, and the changes in the antigen sequence of the influenza viruses were confirmed by gene sequencing and protein structure analysis.

Results: In term of the hemagglutination titer of influenza virus, the reactivity to chicken and guinea pig red blood cell showed different results between egg propagated and cell propagated viruses. In the sequence analysis results for hemagglutinin and neuraminidase, no antigenic mutation was observed throughout all passages when cultured in MDCK-Sky3851 cells. On the other hand, mutations occurred in three amino acid sequences (H156R, G186S, S219F) in hemagglutinin up to 15 passages when cultured in eggs.

Conclusion: H3N2 influenza virus cultured in eggs could lead mutations in amino acid sequence of hemagglutinin, distinct from the corresponding virus cultured in cells for which no antigenic mutation was observed. These findings suggest that cell culture is a more stable and effective way of production with lower risk of antigenic mutations for the manufacture of influenza vaccines.

Keywords: Egg adaptation, Influenza hemagglutinin, Antigenic mutation, Cell culture influenza vaccine

\section{Introduction}

Influenza virus is an infectious virus that infects $5 \%-15 \%$ of the global population every year, causing serious respiratory illnesses and resulting in about half million of deaths [1]. Vaccination is the most effective way to prevent the disease by influenza virus infection. Since influenza viruses continue to evolve by antigenic drift as RNA viruses, World Health Organization updates influenza vaccine components twice a year to match antigenicity with the circulating viruses [2]. Recently, influenza vaccines are mainly quadrivalent vaccines containing two A type (H1N1 and H3N2) and two B type ( $\mathrm{B} /$ Yamagata and $\mathrm{B} /$ Victoria lineage) viruses and most of them are manufactured by 
growing influenza viruses using eggs in a traditional manner. However, propagating the influenza virus in the egg can produce virus variants that are different from those cultured in animal cells, resulting in changed antigenicity from the original virus derived from the patient [3-5]. In particular, candidate vaccine virus (CVV) for vaccine production is mainly produced through passaged culture in eggs, where the antigenicity change may occur due to egg adaption of the virus. This adaptation is the result of several amino acid mutations in hemagglutinin protein. Especially, since the amino acid in which the mutation occurs is a receptor binding site of the hemagglutination (HA) protein or a position very close thereto, the amino acid sequence change at this position may lead to a change in antigenicity $[6,7]$.

Since the human case was confirmed in 1968, H3N2 virus has been more genetically and antigenically evolved than other types of virus so that the vaccine component was updated more than 30 times [8-10]. In particular, low vaccine effectiveness were identified for H3N2 virus in the last 20122013 season, which was reported to be due to the mutation of HA protein by egg adaptations in CVV used in vaccine manufacturing rather than to the antigenic drift of virus [11]. At that time, the CVV for A/Victoria/361/2011 (H3N2) was subjected to amino acid mutations in three position of HA protein by egg adaptations, which were all areas corresponding to the antigenic site as H156Q, G186V, and S219Y. All of these amino acid mutations had increased effects on $\alpha 2,3$-linked receptors and decreased on $\alpha 2,6$-linked receptors in terms of binding avidity. Especially in the case of G186V, it is known to be a mutation that is characteristically found in the egg adaption of the influenza virus [12,13]. The low vaccine effectiveness of H3N2 was also reported in the 2016-2017 season, which is also due to the change of glycosylation site depending on amino acid mutation of HA antigen by egg adaptation $[14,15]$. In the case of clade 3C.2a virus recommended as a vaccine strain, a glycosylation site was added according to the K160T amino acid mutation. But egg-adapted vaccine strain of 3C.2a lacked this glycosylation site, and thus the vaccine effectiveness was lowered even though it was matched well with the actual circulation strain [16]. The correlation between glycosylation and antigenicity of HA protein is well known and influenza virus can avoid host immune system by adding $\mathrm{N}$-linked glycosylation site to HA [17]. H3N2 viruses have evolved more actively through mutations in the antigenic sites of HA proteins, and these amino acid mutations result in the addition of new glycan, which significantly affects antibody binding.
Over the last 50 years, more than 10 new $\mathrm{N}$-glycosylation sites have been added to the H3N2 viral HA antigen, and the addition of these sites has particularly affected the activity of receptor binding site [18]. A meta-analysis of the correlation between influenza virus egg adaptation and vaccine effectiveness showed that egg adaptation has a strong negative correlation with vaccine effectiveness [19]. In other words, passage adaptation in eggs was found to be a strong contribution to the low vaccine effectiveness against H3N2 [20].

Unlike egg, it is known that antigenic mutations do not occur when influenza virus is cultured in animal cells [21-23]. As such, the development of influenza vaccines production systems using Vero or MDCK cells has emerged as a way to resolve the problems pertaining to the egg adaption, and several cell culture influenza vaccines are currently available in the market. Also there are several reports indicating that cell culture influenza vaccine actually showed greater effectiveness than that of egg based vaccine [24-26]. Therefore, it is expected that there will be an increasing interest in cell culture-based production of CVVs and influenza vaccine in the future.

In this study, H3N2 virus was incubated 15 times in animal cells or eggs to confirm and compare the presence of amino acid mutations in hemagglutinin and neuraminidase proteins. Prototype H3N2 virus (A/Texas/50/2012) with no history of subculture in eggs was obtained from the Victorian Infectious Diseases Reference Laboratory (VIDRL) for this study and this virus was propagated using both specific pathogen free (SPF) egg and MDCK-Sky3851 cell line which is the qualified cell lines used in cell culture influenza vaccine production, and the presence or absence of mutation in the antigen sequence of the influenza virus collected at each passage was confirmed.

\section{Materials and Methods}

\section{Virus, embryonated chicken eggs and cells}

Prototype A/Texas/50/2012 (H3N2) influenza virus was obtained from VIDRL (Melbourne, Australia). SPF embryonated chicken eggs were obtained from Namduk SPF (Icheon, Korea) and incubated for 9-10 days at $37^{\circ} \mathrm{C}$ prior to virus inoculation. MDCK-Sky3851 cell line, developed and used in influenza vaccine manufacturing by SK Bioscience (Seongnam, Korea), were maintained in culture medium without serum at $37^{\circ} \mathrm{C}, 5 \% \mathrm{CO}_{2}$. 


\section{Propagation and passage culture of viruses in MDCK-Sky3851 cells and eggs}

Virus seed stocks for A/Texas/50/2012(H3N2) influenza virus were prepared through propagation of prototype virus in $\mathrm{MD}$ CK-Sky3851 cell line several times and these stocks were used in this study. Virus seed stock was inoculated into MDCKSky3851 cells in spinner flask with two different dilution factors of $10^{7}-10^{9}$ and incubated at $34^{\circ} \mathrm{C}, 5 \% \mathrm{CO}_{2}$, and $80 \mathrm{rpm}$ agitation. After 2 days, HA titer was checked and the virus showing the highest HA titer was harvested as a viral stock and stored at $-70^{\circ} \mathrm{C}$. Then, this viral stock was inoculated to MDCK-Sky3851 cell line again and this process was continued up to 15 passages. Virus seed stock was also inoculated into eggs with three different dilution factors of $10^{2}-10^{4}$ and incubated at $37^{\circ} \mathrm{C}$ for 3 days followed by collecting the allantoic fluid after storing at $4^{\circ} \mathrm{C}$ for overnight. The highest HA titer virus was harvested as an egg passage virus stock and stored at $-70^{\circ} \mathrm{C}$. This egg virus stock was also inoculated into eggs again in order to make next passage stock and this process was repeated up to 15 passages.

\section{Hemagglutination assay}

Samples were 2-fold diluted in v-bottomed 96-well microplates and chicken red blood cells at a concentration of $0.5 \%$ were added to each well, and the plates were incubated for 40-50 minutes at room temperature. When the $0.75 \%$ guinea pig red blood cells were used, samples were diluted in roundbottomed 96-well microplates and incubate for 1 hour at room temperature. The sedimentation of red blood cells was evaluated and HA titers were reported as hemagglutinin units (HAU) / $50 \mu \mathrm{L}$.

\section{Gene sequencing and protein structure analysis}

Viral RNA was extracted from each cell and egg passage viruses by using extraction kit (Invitrogen, Carlsbard, CA, USA). Reverse transcription polymerase chain reaction was performed with suitable primer to obtain DNA of hemagglutinin and neuraminidase. Nucleotide sequencing analysis was conducted by Macrogen Inc. (Seoul, Korea) and Geneious software (ver. 10.2.3; Biomatters Ltd., Auckland, New Zealand) was used for data analysis and comparison. Protein structure analysis was conducted by using Phyre2 (ver. 2.0; Structural Bioinformatics Group at Imperial College, London, UK) and UCSF-Chimera software (Resource for Biocomputing, Visualization, and Informatics, San Francisco, CA, USA).

\section{Results}

\section{HA titer for each passage viruses in cells and eggs}

A total of 15 passages in MDCK-Sky3851 cells and eggs were carried out using the prototype A/Texas/50/2012 virus distributed by VIDRL and HA assay was conducted to check the HA titer for viruses of each passage. In case of MDCK-Sky3851 cells, when the chicken red blood cells were used in HA assay, HA titer was identified only in the 1st passage virus $\left(2^{4} \mathrm{HAU} /\right.$ $50 \mu \mathrm{L}$ ) and no titers were showed in all other viruses from the 2nd passage. In contrast, when guinea pig red blood cells were

Table 1. HA titer of H3N2 influenza viruses passaged in MDCK-Sky3851 cells and eggs

\begin{tabular}{|c|c|c|c|c|}
\hline \multirow[b]{2}{*}{ Substrate } & \multirow{2}{*}{$\begin{array}{c}\text { Passage } \\
\text { no. }\end{array}$} & \multirow{2}{*}{$\begin{array}{l}\text { Dilution } \\
\text { factor for } \\
\text { harvest }\end{array}$} & \multicolumn{2}{|c|}{ HA titer (HAU/50 $\mu \mathrm{L})$} \\
\hline & & & $\begin{array}{c}0.5 \% \text { chicken } \\
\text { RBC }\end{array}$ & $\begin{array}{c}0.75 \% \text { Guinea } \\
\text { pig RBC }\end{array}$ \\
\hline \multirow{15}{*}{$\begin{array}{l}\text { MDCK-Sky } \\
3851 \text { cells }\end{array}$} & 1 & $10^{2}$ & $2^{4}$ & $2^{11}$ \\
\hline & 2 & $10^{4}$ & 0 & $2^{11}$ \\
\hline & 3 & $10^{4}$ & 0 & $2^{11}$ \\
\hline & 4 & $10^{5}$ & 0 & $2^{11}$ \\
\hline & 5 & $10^{6}$ & 0 & $2^{11}$ \\
\hline & 6 & $10^{8}$ & 0 & $2^{11}$ \\
\hline & 7 & $10^{8}$ & 0 & $2^{11}$ \\
\hline & 8 & $10^{8}$ & 0 & $2^{11}$ \\
\hline & 9 & $10^{8}$ & 0 & $2^{11}$ \\
\hline & 10 & $10^{8}$ & 0 & $2^{11}$ \\
\hline & 11 & $10^{8}$ & 0 & $2^{11}$ \\
\hline & 12 & $10^{8}$ & 0 & $2^{10}$ \\
\hline & 13 & $10^{8}$ & 0 & $2^{11}$ \\
\hline & 14 & $10^{8}$ & 0 & $2^{11}$ \\
\hline & 15 & $10^{8}$ & 0 & $2^{11}$ \\
\hline \multirow[t]{15}{*}{ Eggs } & 1 & 10 & 0 & 0 \\
\hline & 2 & 1 & 0 & 0 \\
\hline & 3 & 1 & $2^{5}$ & $2^{6}$ \\
\hline & 4 & $10^{2}$ & $2^{5}$ & $2^{5}$ \\
\hline & 5 & $10^{3}$ & $2^{4}$ & $2^{5}$ \\
\hline & 6 & $10^{3}$ & $2^{4}$ & $2^{5}$ \\
\hline & 7 & $10^{3}$ & $2^{4}$ & $2^{5}$ \\
\hline & 8 & $10^{3}$ & $2^{7}$ & $2^{6}$ \\
\hline & 9 & $10^{3}$ & $2^{5}$ & $2^{6}$ \\
\hline & 10 & $10^{4}$ & $2^{5}$ & $2^{7}$ \\
\hline & 11 & $10^{4}$ & $2^{5}$ & $2^{7}$ \\
\hline & 12 & $10^{4}$ & $2^{6}$ & $2^{7}$ \\
\hline & 13 & $10^{6}$ & $2^{4}$ & $2^{5}$ \\
\hline & 14 & $10^{6}$ & $2^{5}$ & $2^{6}$ \\
\hline & 15 & $10^{7}$ & $2^{6}$ & $2^{6}$ \\
\hline
\end{tabular}

$H A$, hemagglutination; HAU, hemagglutinin units; $\mathrm{RBC}$, red blood cell. 
Yong Wook Park et al • Antigenic mutation of influenza virus in egg

used for HA assay, overall HA titer were maintained at $2^{10}-2^{11}$ $\mathrm{HAU} / 50 \mu \mathrm{L}$ with no significant differences among the 1st to 15th passages and no increase in HA titer was also observed as the passage progressed (Table 1). Unlike in the MDCKSky3851 cells, viruses passaged in eggs showed reactivity in both red blood cells up to the 15th passage. Slightly higher HA titers were found in guinea pig red blood cells compared to those of chicken red blood cells from the 3rd passage to 15th passage, $2^{4}-2^{7} \mathrm{HAU} / 50 \mu \mathrm{L}$ in chicken red blood cells and $2^{5}-<2^{8} \mathrm{HAU} / 50 \mu \mathrm{L}$ in guinea pig red blood cells. HA titers were not found in the 1st-2nd passage viruses in both erythrocytes (Table 1).

\section{Gene sequence analysis for hemagglutinin and neuraminidase} of influenza viruses

The hemagglutinin and neuraminidase gene sequences of each passaged virus were compared with those of parent virus. For the sequence of parent viruses, the sequence (ID no. EPI_ISL_122006) registered in Global Initiative on Sharing All Influenza Data was referred to. In the result of sequencing analysis, it was confirmed that hemagglutinin and neuraminidase sequences were $100 \%$ identical to those of parent virus in all passages through the 1st to 15th in MDCK-Sky3851 cells

Table 2. Sequence mutations of hemagglutinin and neuraminidase for each virus passaged in MDCK-Sky3851 cells and eggs

\begin{tabular}{|c|c|c|c|c|}
\hline \multirow[b]{2}{*}{$\begin{array}{l}\text { Passage } \\
\text { no. }\end{array}$} & \multicolumn{2}{|c|}{ MDCK-Sky3851 cells } & \multicolumn{2}{|l|}{ Eggs } \\
\hline & $\begin{array}{l}\text { Hemagglu- } \\
\text { tinin } \\
\text { sequence }\end{array}$ & $\begin{array}{l}\text { Neurami- } \\
\text { nidase } \\
\text { sequence }\end{array}$ & $\begin{array}{l}\text { Hemagglutinin } \\
\text { sequence }\end{array}$ & $\begin{array}{c}\text { Neurami- } \\
\text { nidase } \\
\text { sequence }\end{array}$ \\
\hline 1 & - & - & - & NA \\
\hline 2 & - & - & - & NA \\
\hline 3 & - & - & A515G (H156R) & - \\
\hline 4 & - & - & G604A (G186S) & - \\
\hline 5 & - & - & \multirow[t]{4}{*}{ C942T (silent mutation) } & - \\
\hline 6 & - & - & & - \\
\hline 7 & - & - & & - \\
\hline 8 & - & - & & - \\
\hline 9 & - & - & A515G (H156R) & - \\
\hline 10 & - & - & G604A (G186S) & - \\
\hline 11 & - & - & C704T (S219F) & - \\
\hline 12 & - & - & \multirow[t]{4}{*}{ C942T (silent mutation) } & - \\
\hline 13 & - & - & & - \\
\hline 14 & - & - & & - \\
\hline 15 & - & - & & - \\
\hline
\end{tabular}

"-": There are no differences with parent virus. NA, not available.
(Table 2). In case of viruses passaged in eggs, hemagglutinin sequence of the 1st to 2nd passage viruses was the same as that of the parent virus, but there were three mutations identified from the 3rd to 8th passage viruses, A515G, G604A, and C942T, and from the 9th passaged virus, additional hemagglutinin sequence mutation with $\mathrm{C} 704 \mathrm{~T}$ were detected (Table 2). For the neuraminidase sequence, it was confirmed that the 3 rd to 15 th passage viruses all match $100 \%$ of the sequence of the parent virus.

\section{Protein structure analysis of hemagglutinin}

In the gene sequence analysis, four mutations were identified in hemagglutinin during 15 passage culture in eggs and the effect of these mutations on the amino acid sequences was examined. As a result of codon sequence analysis, it was confirmed that three out of four changes in nucleotide sequence resulted in changes in the amino acid sequence and the other one showed silent mutation (Table 2). In order to determine where these amino acid mutations are located and how these structurally affect the receptor binding sites of the hemagglutinin antigen, the structure analysis was performed using $\mathrm{Ph}$ yre2 software. The analysis revealed that the G186S mutation is relatively inward of the hemagglutinin, while the H156R and S219F mutations are located near the surface of receptor binding site and may cause structural changes (Fig. 1).

\section{Discussion}

Mismatch between circulating influenza viruses and vaccine strains cause a reduction of vaccine effectiveness and this phenomenon is known to be associated with the adaptation in eggs during the manufacturing process of the reassortant CVVs $[27,28]$. When the influenza virus is cultured in eggs, antigenic mutations may be caused by egg adaptation, and it is reported that this phenomenon is particularly prominent in H3N2 virus $[3,6]$. In this study, in order to confirm the mutations due to egg adaptation of influenza virus, prototype A/ Texas/50/2012 virus that had not been passaged in eggs were obtained from VIDRL and the virus was passaged up to 15 times in MDCK-Sky3851 cells, which are Skycellflu vaccine producing cell lines, and in embryonated eggs, respectively, and then HA titer and gene sequences of hemagglutinin and neuraminidase were compared.

The HA titer of the prototype virus prior to the 1st passage is $2^{4} \mathrm{HAU} / 50 \mu \mathrm{L}$ for both chicken and guinea pig red blood cells, and when this virus was passaged in MDCK-Sky3851 

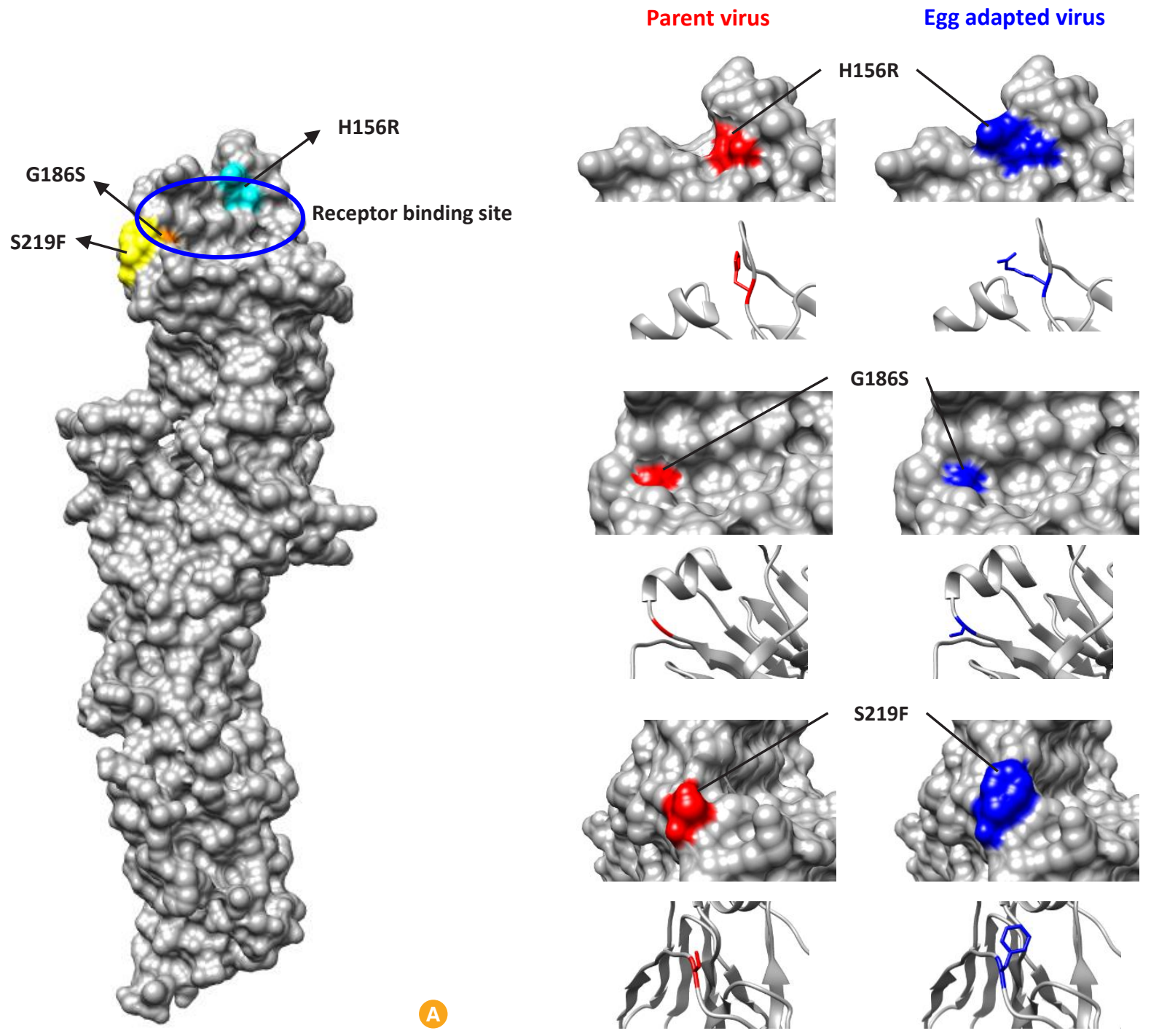

G186S
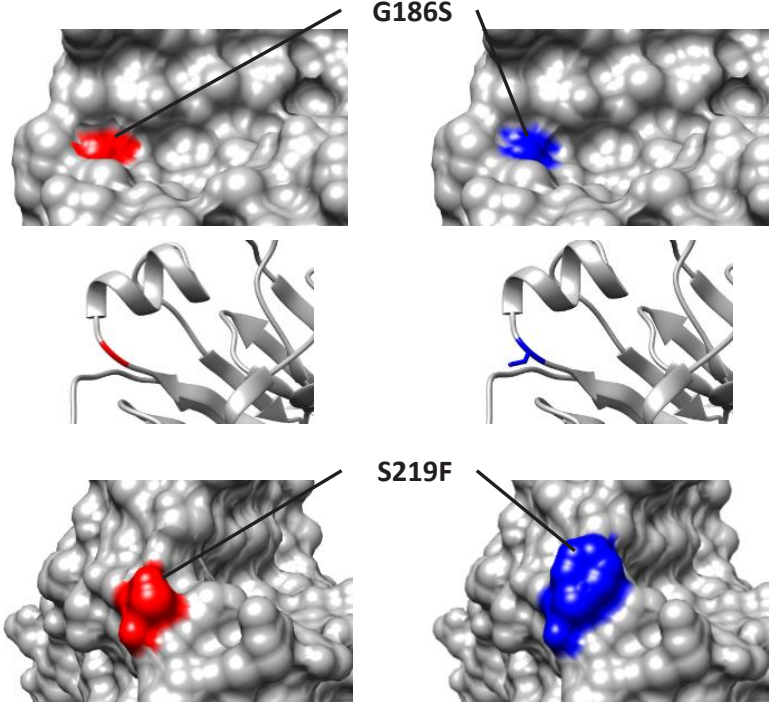

S219F
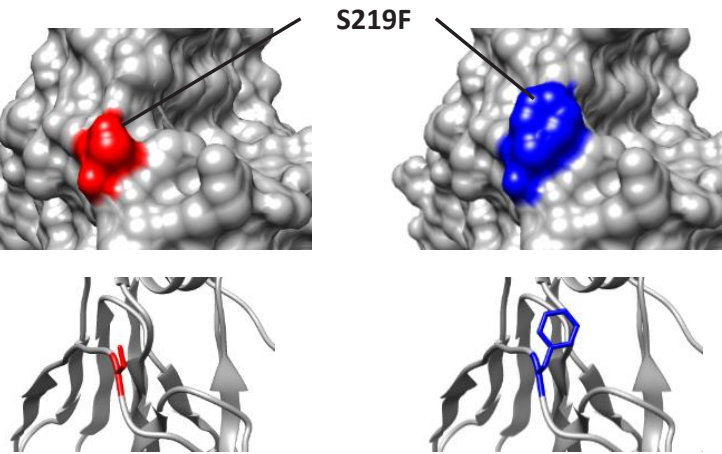

Fig. 1. Structure of hemagglutinin and mutation points of amino acid during egg cultivation of H3N2 influenza virus (A/Texas/50/2012). (A) All three mutated amino acid were located near the receptor binding site of hemagglutinin. (B) Detail structures were compared for mutated amino acid during egg cultivation. Red color shows the original hemagglutinin structure of parent virus and blue color shows the mutated structure by egg adaptation. Surface view and backbone structure showing corresponding amino acid residue were presented for each mutation.

cells, it was confirmed that the HA titer increased up to $2^{11}$ $\mathrm{HAU} / 50 \mu \mathrm{L}$ from the first passage in the guinea pig red blood cells which corresponding to 128 times increase compared to the prototype virus and this was maintained up to the 15th passages. However, HA titer was not detected from the 2nd passage in chicken red blood cells, and it was confirmed that the reactivity of virus may be lowered with chicken red blood cells when passaged in MDCK-Sky3851 cells. In case of egg culture virus, HA titer was not determined in both chicken and guinea pig red blood cells until the 2nd passage, and HA titer began to appear at $2^{5} \mathrm{HAU} / 50 \mu \mathrm{L}$ in chicken red blood cells and $2^{6} \mathrm{HAU} / 50 \mu \mathrm{L}$ in guinea pig red blood cells from the 3rd passage. Until the 15th passages, HA titers were maintained quite constantly, not showing much variability, with a maximum of $2^{7} \mathrm{HAU} / 50 \mu \mathrm{L}$ in chicken red blood cells and a maximum of $2^{8} \mathrm{HAU} / 50 \mu \mathrm{L}$ in guinea pig red blood cells. Based on the HA assay results using chicken and guinea pig red blood cells, it was confirmed that the binding avidity of influenza virus varies among the different types of red blood cells depending upon the host species and the substrate for passage of influenza virus. Since the prototype virus used in this study was originated from human, it would be highly reactive to $\alpha 2,6$ linked receptors. Thus, when the virus was cultured in MDCKSky3851 cells, the virus was responsive to guinea pig red blood cells which contain $\alpha 2,6$-linked receptors as it is of the same mammalian origin, but not to chicken red blood cells with 
the $\alpha 2,3$-linked receptors as it is of avian origin. In contrast, when the prototype virus was cultured in egg, it did not grow well with no confirmed HA titer until the 2nd passages since it was not the host species from which the virus had been originally propagated. From the 3rd passage, it seems that the virus became capable to respond to both types of chicken and guinea pig red blood cells as they propagated and adapted to the egg. This suggests that the virus has binding avidity for both $\alpha 2,3$ and $\alpha 2,6$-linked receptors. However HA titer with guinea pig red blood cells was lower for egg cultured virus, compared to that of MDCK-Sky3851 cell cultured virus, suggesting that it may be owing to the egg adaptation.

Subsequently, the nucleotide sequences of the hemagglutinin and neuraminidase genes, which are antigens located on the surface of virus, were analyzed to determine if the antigenicity of the viruses passaged 15 times in MDCK-Sky3851 cells and eggs was altered. As a result, it was confirmed that the nucleotide sequences of the viruses from MDCK-Sky3851 cells were $100 \%$ identical to those of the parent virus across all passages from the 1st to 15th without any mutation on both hemagglutinin and neuraminidase sequences. In the case of egg cultured viruses, no mutation was observed in the sequence of the hemagglutinin gene up to the 2nd passage, but there were three changes identified for A516G, G604A, and $\mathrm{C} 942 \mathrm{~T}$ in the 3rd to 8th passaged viruses, and the corresponding amino acid sequence mutations were H156R, G186S, and silent mutation, respectively. In addition, the C704T mutation was further identified in the 9th to 15th passaged viruses, and the corresponding amino acid sequence mutation was identified as S219F. It was confirmed that these mutations are identical to the amino acid positions $(156,186,219)$ that caused antigenic mutations in IVR-165, the H3N2 vaccine strain of the 2012-2013 Northern Hemisphere season, which had low vaccine effectiveness due to antigenic mismatch between the circulating virus and vaccine strain [11]. In the result of neuraminidase gene sequencing analysis of egg cultured viruses, it was confirmed that the 3rd to 15th passages were $100 \%$ identical in sequence to those of the parent virus, except for the 1st and 2nd passages which were not sequenced. As the 1st and 2nd passaged viruses had HA titer of $0 \mathrm{HAU} / 50$ $\mu \mathrm{L}$, the sequence of neuraminidase could not be confirmed by polymerase chain reaction due to low virus contents. However, since no mutations were identified in the neuraminidase gene sequences of the 3rd to 15th passaged viruses, it is expected that the neuraminidase gene sequences of the 1st and 2nd stocks also may not carry any mutations.
In the viruses cultured in egg, a total of three amino acid mutations were confirmed, and a structural analysis was performed to confirm what structural and functional changes these may induce in hemagglutinin antigen. Influenza hemagglutinin has five antigenic sites [29-32], among which antigenic site $\mathrm{A}$ and $\mathrm{B}$ are located at the distal end from the virus surface and contain receptor binding sites, in particular antigenic site B is known as the immonodominant [33]. The hemagglutinin receptor binding site is composed of 130-loop, 150loop, 190-helix, and 220-loop structures, forming a concave shape, and the sialic acid is bound here [34]. Influenza viruses have evolved naturally through mutations in receptor binding sites to evade antibody responses from the host. Therefore, amino acid mutations in the position that constitutes the receptor binding site can lead to changes in viral antigenicity through structural changes. In this study, three amino acid mutations were identified in the influenza virus cultured in egg, including H156R, G186S, and S219F. In terms of amino acid position, all of the amino acid mutations were very close to the position of the structure constituting the hemagglutinin receptor binding site, so it can be seen that there is a possibility of inducing changes in receptor binding avidity. According to the structural analysis of the hemagglutinin, the structure before and after mutation was quite different at position 156 where histidine was changed to arginine and 219 where serine was changed to phenylalanine. On the other hand, the change of glutamic acid to serine at amino acid position 186 was not significant in the structural difference (Fig. 1). G186S and H156R are mutations that normally occur characteristically in egg adaptation, and show the effect of improving replication by increasing the binding avidity of $\alpha 2,3-$ linked receptors rather than $\alpha 2,6$-linked receptors. However, G186S is known to show an insignificant effect in terms of antigenicity $[3,35]$. S219F mutation also affects binding avidity, especially in the case of phenylalanine, which is an amino acid containing a bulky aromatic ring, which may affect 220-loop conformation and physically alter the receptor binding site [3]. This can lead to changes in antigenicity, and it is also known to act synergistically to change the antigenicity of influenza virus, especially when positions 186 and 219 change simultaneously [3]. Thus, all three amino acid mutations identified in this study were related to egg adaptation and were identified as the same positions as amino acid mutations known to affect antigenic changes which result in low vaccine effectiveness of influenza viruses. In addition to these results, it is necessary to conduct animal test to determine 
whether amino acid mutations are directly related to actual antigenic changes. It would be desirable to confirm the correlation between the incidence of mutation and the actual vaccine efficacy through animal immunization and challenge test using the mutated virus in egg and the non-mutated virus in cell.

In conclusion, it was confirmed that when the H3N2 influenza virus was cultured in egg, amino acid mutations occurred near the receptor binding site of hemagglutinin, which may alter binding avidity and antigenicity accordingly. On the other hand, it was also confirmed that such amino acid mutations did not occur at all when the influenza virus is cultured in MDCK-Sky3851 cells. Therefore, cell culture influenza vaccines production system is a more efficient and reliable method for producing vaccines in that influenza vaccines can be produced without the risk of antigenic changes from the actual prevalent influenza virus. In this respect, the importance of the cell culture influenza vaccine is anticipated to increase further, and it is essential to continuously monitor and evaluate the change of vaccine effectiveness due to amino acid mutation in egg cultured vaccines.

\section{ORCID}

Yong Wook Park https://orcid.org/0000-0003-1198-7835

Yun Hee Kim https://orcid.org/0000-0001-7496-808X Hwan Ui Jung https://orcid.org/0000-0003-3392-0885

Oh Seok Jeong https://orcid.org/0000-0002-6465-9387

Eun Ji Hong https://orcid.org/0000-0002-9035-1597

Hun Kim https://orcid.org/0000-0003-3726-7094

Jae Il Lee https://orcid.org/0000-0003-0236-3297

\section{References}

1. Vemula SV, Zhao J, Liu J, Wang X, Biswas S, Hewlett I. Current approaches for diagnosis of influenza virus infections in humans. Viruses 2016;8:96.

2. Barr IG, Russell C, Besselaar TG, et al. WHO recommendations for the viruses used in the 2013-2014 Northern Hemisphere influenza vaccine: epidemiology, antigenic and genetic characteristics of influenza A(H1N1)pdm09, A(H3N2) and B influenza viruses collected from October 2012 to January 2013. Vaccine 2014;32:4713-25.

3. Parker L, Wharton SA, Martin SR, et al. Effects of egg-adaptation on receptor-binding and antigenic properties of recent influenza A (H3N2) vaccine viruses. J Gen Virol
2016;97:1333-44.

4. Gambaryan AS, Robertson JS, Matrosovich MN. Effects of egg-adaptation on the receptor-binding properties of human influenza A and B viruses. Virology 1999;258:232-9.

5. Raymond DD, Stewart SM, Lee J, et al. Influenza immunization elicits antibodies specific for an egg-adapted vaccine strain. Nat Med 2016;22:1465-9.

6. Meyer WJ, Wood JM, Major D, Robertson JS, Webster RG, Katz JM. Influence of host cell-mediated variation on the international surveillance of influenza A (H3N2) viruses. Virology 1993;196:130-7.

7. Kodihalli S, Justewicz DM, Gubareva LV, Webster RG. Selection of a single amino acid substitution in the hemagglutinin molecule by chicken eggs can render influenza A virus (H3) candidate vaccine ineffective. J Virol 1995;69: 4888-97.

8. Russell CA, Jones TC, Barr IG, et al. The global circulation of seasonal influenza A (H3N2) viruses. Science 2008;320: 340-6.

9. Russell CA, Jones TC, Barr IG, et al. Influenza vaccine strain selection and recent studies on the global migration of seasonal influenza viruses. Vaccine 2008;26 Suppl 4:D31-4.

10. Neher RA, Bedford T, Daniels RS, Russell CA, Shraiman BI. Prediction, dynamics, and visualization of antigenic phenotypes of seasonal influenza viruses. Proc Natl Acad Sci U S A 2016;113:E1701-9.

11. Skowronski DM, Janjua NZ, De Serres G, et al. Low 201213 influenza vaccine effectiveness associated with mutation in the egg-adapted H3N2 vaccine strain not antigenic drift in circulating viruses. PLoS One 2014;9:e92153.

12. Chen Z, Zhou H, Jin H. The impact of key amino acid substitutions in the hemagglutinin of influenza A (H3N2) viruses on vaccine production and antibody response. Vaccine 2010;28:4079-85.

13. Barman S, Franks J, Turner JC, Yoon SW, Webster RG, Webby RJ. Egg-adaptive mutations in H3N2v vaccine virus enhance egg-based production without loss of antigenicity or immunogenicity. Vaccine 2015;33:3186-92.

14. Melidou A, Gioula G, Exindari M, et al. Influenza A(H3N2) genetic variants in vaccinated patients in northern Greece. J Clin Virol 2017;94:29-32.

15. Flannery B, Chung JR, Thaker SN, et al. Interim estimates of 2016-17 seasonal influenza vaccine effectiveness: United States, February 2017. MMWR Morb Mortal Wkly Rep 2017;66:167-71.

16. Zost SJ, Parkhouse K, Gumina ME, et al. Contemporary 
H3N2 influenza viruses have a glycosylation site that alters binding of antibodies elicited by egg-adapted vaccine strains. Proc Natl Acad Sci U S A 2017;114:12578-83.

17. Zhang X, Chen S, Jiang Y, et al. Hemagglutinin glycosylation modulates the pathogenicity and antigenicity of the H5N1 avian influenza virus. Vet Microbiol 2015;175:24456.

18. Wu NC, Wilson IA. A Perspective on the structural and functional constraints for immune evasion: insights from influenza virus. J Mol Biol 2017;429:2694-709.

19. Belongia EA, Simpson MD, King JP, et al. Variable influenza vaccine effectiveness by subtype: a systematic review and meta-analysis of test-negative design studies. Lancet Infect Dis 2016;16:942-51.

20. Chen H, Alvarez JJS, Ng SH, Nielsen R, Zhai W. Passage adaptation correlates with the reduced efficacy of the influenza vaccine. Clin Infect Dis 2019;69:1198-204.

21. Katz JM, Wang M, Webster RG. Direct sequencing of the HA gene of influenza (H3N2) virus in original clinical samples reveals sequence identity with mammalian cell-grown virus. J Virol 1990;64:1808-11.

22. Robertson JS, Nicolson C, Bootman JS, Major D, Robertson EW, Wood JM. Sequence analysis of the haemagglutinin (HA) of influenza A (H1N1) viruses present in clinical material and comparison with the HA of laboratory-derived virus. J Gen Virol 1991;72:2671-7.

23. Ito T, Suzuki Y, Takada A, et al. Differences in sialic acidgalactose linkages in the chicken egg amnion and allantois influence human influenza virus receptor specificity and variant selection. J Virol 1997;71:3357-62.

24. Barr IG, Donis RO, Katz JM, et al. Cell culture-derived influenza vaccines in the severe 2017-2018 epidemic season: a step towards improved influenza vaccine effectiveness. NPJ Vaccines 2018;3:44.

25. Izurieta HS, Chillarige Y, Kelman J, et al. Relative effectiveness of cell-cultured and egg-based influenza vaccines among elderly persons in the United States, 2017-2018. J Infect Dis 2019;220:1255-64.
26. Flannery B, Fry AM. Comparing influenza vaccine types: the path toward improved influenza vaccine strategies. J Infect Dis 2019;220:1237-9.

27. Chan MCW, Wang MH, Chen Z, et al. Frequent genetic mismatch between vaccine strains and circulating seasonal influenza viruses, Hong Kong, China, 1996-2012. Emerg Infect Dis 2018;24:1825-34.

28. Heikkinen T, Ikonen N, Ziegler T. Impact of influenza B lineage-level mismatch between trivalent seasonal influenza vaccines and circulating viruses, 1999-2012. Clin Infect Dis 2014;59:1519-24.

29. Wiley DC, Skehel JJ. The structure and function of the hemagglutinin membrane glycoprotein of influenza virus. Annu Rev Biochem 1987;56:365-94.

30. Gerhard W, Webster RG. Antigenic drift in influenza A viruses. I. Selection and characterization of antigenic variants of $\mathrm{A} / \mathrm{PR} / 8 / 34$ (HON1) influenza virus with monoclonal antibodies. J Exp Med 1978;148:383-92.

31. Webster RG, Laver WG. Determination of the number of nonoverlapping antigenic areas on Hong Kong (H3N2) influenza virus hemagglutinin with monoclonal antibodies and the selection of variants with potential epidemiological significance. Virology 1980;104:139-48.

32. Skehel JJ, Stevens DJ, Daniels RS, et al. A carbohydrate side chain on hemagglutinins of Hong Kong influenza viruses inhibits recognition by a monoclonal antibody. Proc Natl Acad Sci U S A 1984;81:1779-83.

33. Popova L, Smith K, West AH, et al. Immunodominance of antigenic site B over site A of hemagglutinin of recent H3N2 influenza viruses. PLoS One 2012;7:e41895.

34. Lin YP, Xiong X, Wharton SA, et al. Evolution of the receptor binding properties of the influenza A(H3N2) hemagglutinin. Proc Natl Acad Sci U S A 2012;109:21474-9.

35. Lu B, Zhou H, Chan W, Kemble G, Jin H. Single amino acid substitutions in the hemagglutinin of influenza A/Singapore/21/04 (H3N2) increase virus growth in embryonated chicken eggs. Vaccine 2006;24:6691-3. 